\title{
Adrenocortical neoplasia: evolving concepts in tumorigenesis with an emphasis on adrenal cortical carcinoma variants
}

\author{
Ronald R. de Krijger • Thomas G. Papathomas
}

Received: 6 September 2011 /Revised: 28 September 2011 /Accepted: 31 October 2011 /Published online: 16 November 2011

(C) The Author(s) 2011. This article is published with open access at Springerlink.com

\begin{abstract}
Adrenocortical carcinoma (ACC) is a rare, heterogeneous malignancy with a poor prognosis. According to WHO classification 2004, ACC variants include oncocytic ACCs, myxoid ACCs and ACCs with sarcomatous areas. Herein, we provide a comprehensive review of these rare subtypes of adrenocortical malignancy and emphasize their clinicopathological features with the aim of elucidating aspects of diagnostic categorization, differential diagnostics and biological behavior. The issue of current terminology, applied to biphasic tumors with pleomorphic, sarcomatous or sarcomatoid elements arising in adrenal cortex, is also discussed. We additionally present emerging evidence concerning the adrenal cortical tumorigenesis and the putative adenoma-carcinoma sequence as well.
\end{abstract}

Keywords Adrenal cortical neoplasia - Adenoma . Carcinoma ACC variants - Oncocytic · Myxoid .

Sarcomatoid $\cdot$ Carcinosarcoma

\section{Introduction}

Adrenocortical carcinoma (ACC) is a rare heterogeneous malignancy with an incidence of $0.7-2.0$ cases per million population per year [1]. Although the diagnosis of malignancy is easy in most cases, mainly due to advanced stage at

R. R. de Krijger $(\bowtie) \cdot$ T. G. Papathomas

Department of Pathology, Josephine Nefkens Institute, Erasmus MC-University Medical Center Rotterdam, P.O. Box 2040, 3000 CA Rotterdam, The Netherlands e-mail: r.dekrijger@erasmusmc.nl

T. G. Papathomas

e-mail: t.papathomas@erasmusmc.nl presentation, strictly intra-adrenal tumors must be evaluated for malignant potential [2]. In this regard, several multiparametric scoring systems, including the Hough scoring system, the Weiss scoring system, the Van Slooten scoring system and the Weiss revisited index [3, 4], and, also recently, diagnostic algorithms, such as the stepwise discriminate diagnostic system and the simplified diagnostic algorithm [5, 6], have been generated. The latter takes into account the presence of a disrupted reticulin framework, which constitutes the first step of this diagnostic approach, as being the single most sensitive feature of malignancy. Other parameters include mitotic count $>5 / 50 \mathrm{HPF}$, presence of necrosis and venous invasion [6].

Currently, the Weiss scoring system is the most popular among multiparametric scoring systems, owing to its reliability and relative simplicity (Table 1) [1,7]. Nevertheless, the Weiss system suffers particular limitations including (1) lack of reproducibility of several criteria, (2) "borderline" tumors with a Weiss score of 2 or 3, (3-4) oncocytic and myxoid variants of adrenocortical tumors and (5) pediatric adrenocortical neoplasms [2, 7]. The latter are frequently characterized by a limited malignant potential especially in children $<5$ years of age, despite the presence of impressively atypical histologic features (Fig. 1), which could be attributed either to a low stage upon presentation or to a putative origin from a cell of the fetal adrenal cortex [8].

The objectives of this review are (1) to present emerging evidence concerning the adrenocortical tumorigenesis in order to address the issue of an adenoma-carcinoma sequential progression as well as to further elucidate the incompletely understood pathophysiology of this aggressive neoplasia and (2) to describe in detail the rare variants of ACC to increase awareness of their clinicopathological features and the potential diagnostic challenges. 
Table 1 Weiss system for assessing malignant potential of adrenocortical neoplasms $[2,3]$

Histological features to be evaluated

Diffuse architecture (greater than one third of the tumor)

Clear cells comprising $25 \%$ or less of the tumor

High nuclear grade (grade 3 or 4 according to Fuhrman criteria)

Mitotic rate $\geq 6$ per 50 high-power fields

Atypical mitotic figures

Necrosis

Venous invasion

Sinusoidal invasion

Capsular invasion

The presence of three or more criteria highly correlates with malignant behavior

\section{Part I. Histogenesis of conventional adrenocortical carcinomas}

A hotly debated issue is whether or not adenomas progress stepwise to carcinoma. Given (1) the differences between benign and malignant tumors in terms of transcriptome and genetic alterations, (2) the epidemiology of adrenal incidentaloma and ACC and (3) the low incidence of progression $(5-25 \%)$ of clinically inapparent adrenal masses to hyperfunction or an increase in size by at least $1 \mathrm{~cm}$ coupled with (4) the lack of clear evolution from benign, non-secreting adenoma to ACC in untreated cases $[9,10]$, it seems that multistep progression from benign to malignant is unlikely for adrenal cortical neoplasms.

However, not all evidence points in that direction. Fewer genetic aberrations were found in adenomas than in carcinomas. Most (72-86\%) distinct alterations seen in the adenomas were also present in carcinomas [11, 12]. Furthermore, the positive correlation between the number of alterations detected by comparative genomic hybridiza- tion $(\mathrm{CGH})$ and tumor size $[11,13]$ supports the notion of an adenoma-carcinoma sequence. Additional evidence in favor of such a sequence derives from X-chromosome inactivation pattern analysis which shows monoclonal ACCs along with ACAs of polyclonal or monoclonal origin. Allelotyping with microsatellites and a few reported cases provide support for an adrenal adenoma-carcinoma sequence [14-20].

With regard to the latter, Bernard et al. [17] reported on an intriguing case of a localized adrenal neoplasm composed of two different components exhibiting different pathological features: a central zone of a Weiss score (5) and a peripheral zone of a Weiss score (0). The former zone displayed complete LOH at the TP53 (17p13.3) gene locus, uniparental disomy at the $11 \mathrm{p} 15$ locus, overexpression of the IGF-II gene and numerous CGH changes, with gains at chromosomes 4, 5p, 10, 12p, 16p, 19 and 20 and losses at chromosomes 2 and 11 . The outer zone did not show any of these genetic abnormalities. A similar case was presented by Trezzi et al. [18] as a dedifferentiated adrenal cortical neoplasm comprising a sharply outlined, hemorrhagic, central nodule (ACC) and an outer ring zone (ACA), while Gaujoux et al. [19] included one heterogeneous ACT in a small series consisting of an ACC component (Weiss score 6) developed within an ACA component (Weiss score 1). The malignant component exhibited diffuse cytoplasmic and focal nuclear $\beta$-catenin accumulation and harbored a $\beta$ catenin gene (CTNNB1) mutation, whereas the benign component showed only focal cytoplasmic staining and no such mutation. No TP53 somatic mutation was found in the ACC and ACA components. Similarly, Schmitt et al. [20] presented an ACA case showing frank heterogeneity both by means of H\&E staining (atypia, hyperchromasia, nucleoli and atypical mitoses) and immunohistochemistry, including perinuclear dot-like immunoreactivity for IGF-II, nuclear staining for CDK4 and Ki67 labeling index $>5 \%$. These particular areas within the adenoma were interpreted as small

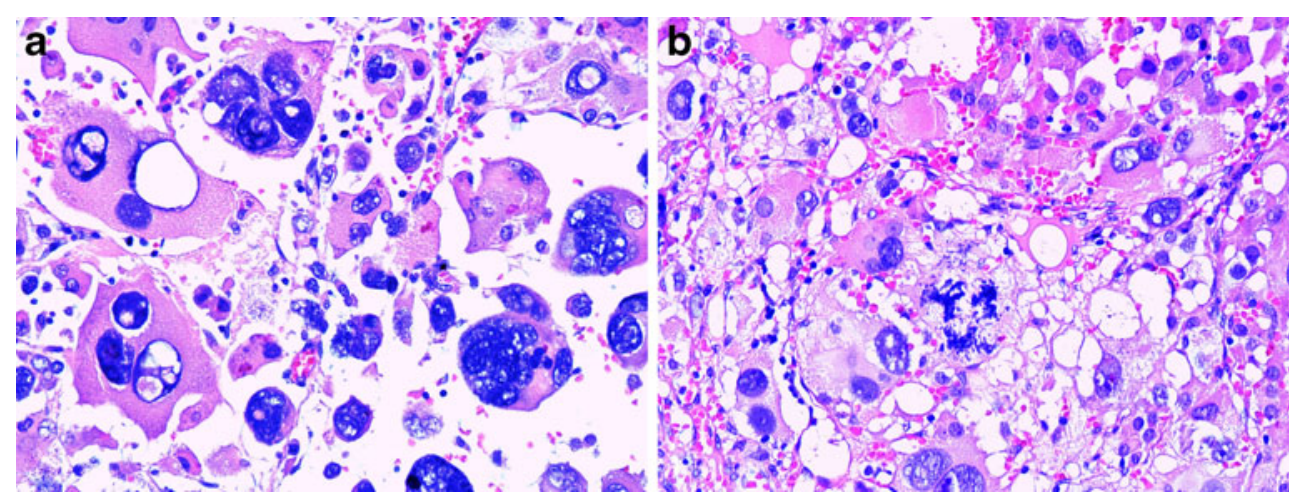

Fig. 1 Two areas from a pediatric adrenal cortical neoplasm, weighing $88 \mathrm{~g}$. Large, multinucleated tumor giant cells, with hyperchromatic nuclei and nuclear pseudoinclusions in a few, among smaller tumor cells displaying high nuclear/cytoplasmic ratio and hyperchromatic nuclei (a). Tumor cells with moderately atypical nuclei and an atypical mitotic figure near the center of the field (b). Despite these worrisome histological features, this neoplasm had a benign biological behavior 
foci of malignant transformation, in accordance with a van Slooten index $>8$.

Whether the fact that different scoring systems yield different diagnoses in individual ACTs [20, 21] points to a multistep process remains to be clarified. It is tempting to speculate that in a process of clonal evolution, one dominant clone may overtake neighboring subclones to an extent that the ACA component is no longer visible.

Recent evidence has suggested multistep tumorigenesis within the group of ACCs [9]. In particular, transcriptome analysis has established an ACC subclassification into two distinct subgroups with different outcomes [22-24], consistent with the notion that ACC survival and outcome vary greatly mainly due to different molecular defects modulating the tumor phenotype and, to a lesser extent, to tumor stage at diagnosis [25]. However, ACCs in the poor prognosis group displayed more advanced disease, subsequently confounding the issue of whether these subgroups correspond to distinct stages of the same disease or to distinct ACC types. Statistical independence of transcriptome-based prognostic information from stage seems to argue in favor of the latter [26].

In an effort to further elucidate the molecular genetics of the poor-outcome ACC group, Ragazzon et al. [25] identified three subgroups by using unsupervised clustering analysis: (1) p53 group, encompassing all tumors with a TP53 mutation, (2) $\beta$-catenin group, containing all tumors with beta-catenin nuclear staining, and (3) x group, with neither p53 nor $\beta$-catenin altered pathways, but enriched in cell cycle and metabolism genes. Moreover, IGF-II overexpression has been shown in approximately $90 \%$ of ACCs, with paternal unidisomy and loss of imprinting being the underlying mechanisms accounting for this markedly elevated expression in familial ACC cases associated with Beckwith-Wiedemann Syndrome, while additional mechanisms of transcriptional regulation are likely to be involved in sporadic ACCs [9, 23]. In this context, Ragazzon et al. [25] did refer to widespread IGF-II overexpression among both subgroups, highlighting its importance in adrenocortical tumorigenesis, but concomitantly suggesting a role for additional genomic alterations promoting the development of the most aggressive tumors [25, 27]. Prior to IGF-II overexpression, it appears that ACC loses nephroblastoma overexpressed (nov) expression [28], in line with the finding of a significantly reduced novH expresssion correlated with the acquisition of a malignant tumoral phenotype [29].

Several lines of evidence suggest that the activation of the $\mathrm{Wnt} / \beta$-catenin signaling pathway is important in adrenocortical oncogenesis [19, 25, 30-37]. In fact, it has been demonstrated that this activation, as assessed by abnormal nuclear and/or cytoplasmic $\beta$-catenin accumulation with or without somatic activating CTNNB1 mutations, is involved both in benign and malignant adrenocortical tumors [19, 31-33]. It is of particular interest to note that CTNNB1 mutations (1) were detected only in macronodules of primary pigmented nodular adrenocortical disease cases, suggesting that these are secondary genetic events contributing to the nodular development of the disease and potentially implying a more aggressive phenotype [19, 34], (2) were related to a specific phenotype of larger and non-secreting ACAs, indicating a less differentiated state [33], and (3) were associated with decreased overall and disease-free survival in ACCs, suggesting a specific effect on tumor biology in terms of progression towards a more aggressive phenotype within the group of ACCs [25, 35]. Altogether, these observations, along with data stemming from an experimental mouse model [30], do not completely clarify whether the activation of Wnt/betacatenin signaling pathway is an early or a late event in the development of adrenal neoplasms.

\section{Part II. Adrenocortical carcinoma variants}

\section{Oncocytic adrenocortical neoplasms}

Oncocytic adrenocortical neoplasms (OAN) represent a rare group of neoplasms with approximately 115 cases reported in the literature [38-44]. Kakimoto et al. [38] first described this particular histological variant in 1986, which was followed by numerous reports either in the form of single cases or of small series, with the largest one comprising 13 cases [39].

Accurate classification of OAN is important. Given that OANs display (1) cells with eosinophilic cytoplasm, clear cell population as a rule consisting of less than $25 \%$ of the tumor volume, (2) high-grade nuclear atypia in at least a subgroup of neoplastic cells and (3) almost always diffuse architecture and since these inherent histological parameters do not confer a poor prognosis [45], applying the Weiss system to this subset of adenocortical neoplasms will lead to a diagnosis of ACC, which is not appropriate given the benign behavior of most of these lesions.

Bisceglia and colleagues [46] developed criteria for the classification of oncocytic adrenocortical tumors (Table 2). These were based on parameters of the Weiss system and constituted the Lin-Weiss-Bisceglia (LWB) system: (1) major criteria (a mitotic rate of more than 5 mitoses per 50 high-power fields, any atypical mitoses or venous invasion), (2) minor criteria [large size (>10 cm and/or > $200 \mathrm{gr}$ ), necrosis, capsular invasion or sinusoidal invasion] and (3) definitional criteria (predominantly cells with eosinophilic-granular cytoplasm, high nuclear grade and diffuse architectural pattern). The latter are not used as they 
Table 2 Lin-Weiss-Bisceglia (LWB) system for diagnostic categorization of oncocytic adrenocortical neoplasms [42]

Major criteria
Mitotic count $>5$ per 50 high-power fields
Atypical mitoses
Venous invasion
Minor criteria
Size $>10 \mathrm{~cm}$ and/or weight $>200 \mathrm{~g}$
Necrosis
Sinusoidal invasion
Capsular invasion

The presence of one major criterion indicates malignancy, one to four minor criteria present indicates uncertain malignant potential, and the absence of all major and minor criteria is indicative of benign biological behavior

are present in all subgroups of pure oncocytic neoplasms and are not determinants of biological behavior. According to the proposed working rules, the presence of any one of the major criteria indicates malignancy (oncocytic adrenocortical carcinoma), and the presence of one to four minor criteria is indicative of uncertain potential (borderline oncocytic neoplasm of uncertain malignant potential) (Fig. 2), while the absence of all major and minor criteria indicates benign behavior (adrenocortical oncocytoma) [46]. In addition, Bisceglia et al. [45] proposed categories for oncocytic adrenocortical tumors as follows: (1) pure oncocytic tumor, if a tumor is exclusively or almost entirely composed (greater than 90\%) of oncocytic cells, (2) mixed oncocytic tumor, when a clear cell component is also present (ranging from $10 \%$ to $50 \%$ ), and (3) ordinary

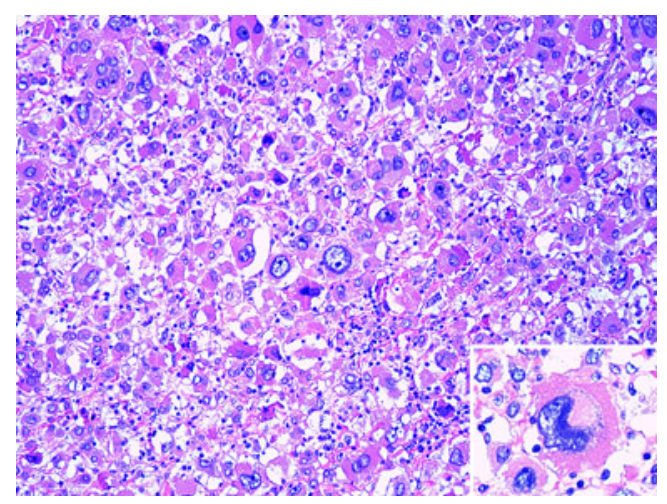

Fig. 2 Section of an oncocytic adrenocortical neoplasm, weighing $>200 \mathrm{~g}$; in the presence of only one minor criterion (according to the Lin-Weiss-Bisceglia classification), a diagnosis of borderline oncocytic neoplasm of uncertain malignant potential was finally rendered. Diffuse growth pattern of tumor cells, characteristically displaying abundant eosinophilic, granular cytoplasm and at least focal high nuclear atypia. Marked degree of nuclear pleomorphism (inset). These would lead to a Weiss score 3, probably overestimating the biological behavior of this lesion adrenocortical tumor with focal oncocytic changes, if the oncocytic component is not a predominant one (less than $50 \%$ of the tumor mass).

It is critical to extensively sample an oncocytic adrenocortical neoplasm for several reasons. First, one should discriminate a pure oncocytic tumor either from an ordinary adrenocortical tumor with focal oncocytic changes or its conventional counterpart of the compact cell type in order not to incorrectly apply the Weiss system and accordingly inadequately estimate its biological behavior [45]. Second, an oncocytic tumor can only be labeled as pure after quantifying each individual component (oncocytic and clear) since pure and mixed oncocytic tumors do not seem to share similar clinical outcome [45]. In this regard, immunohistochemistry using antimitochondrial antibodies can support the recognition of oncocytic differentiation and help to accurately quantify the oncocytic component. Of note, adrenocortical cells are, by nature, rich in mitochondria and therefore positive, so only strong, diffuse and finely granular staining should be taken as indicative of oncocytic differentiation, as suggested by Wong et al. [39] with respect to mES-13 staining. Moreover, the differential diagnostic spectrum of OANs also includes the oncocytic variant of pheochromocytoma, the granular cell variant of clear cell renal cell carcinoma, the eosinophilic variant of chromophobe renal cell carcinoma and the hepatocellular carcinoma [45, 46].

In an effort to further delineate and elucidate this subtype of adrenocortical neoplasia, Wong et al. [39] provided a comprehensive review and retrospectively applied the LWB criteria to all reported cases with sufficient information, along with 13 previously unpublished cases. Of note, they validated the effectiveness of the LWB criteria in predicting malignant potential and provided preliminary evidence supporting the concept of a more favorable prognosis for oncocytic adrenocortical carcinomas in comparison with their conventional counterparts. Moreover, they found approximately $30 \%$ of OANs to be functional while emphasizing the frequency of small oncocytes in several cases of their series [39], accordingly including in their view of the oncocyte not only cells with abundant granular eosinophilic cytoplasm.

\section{Myxoid adrenocortical neoplasms}

In keeping with the current notion that myxoid extracellular matrix is a histological feature that can be found in both physiological and pathological conditions, non-neoplastic (e.g. myxedema) as well as neoplastic (benign/malignant epithelial or mesenchymal tumors) [47], myxoid change has been recognized rarely also in neoplasms of adrenal cortical origin. The myxoid substance has been shown to be Alcianblue positive and negative or focally weak positive for $P A S$ 
stain and mucicarmine. In particular, there have been thus far 46 reports of myxoid adrenocortical neoplasms (MANs) [48-65]: 17 myxoid adrenocortical adenomas [49-58], 2 borderline MANs [48, 50] and 27 myxoid adrenocortical carcinomas [48, 49, 59-65]. These cases were either published as isolated case reports or were presented in small series, with the largest ones each consisting of 14 cases [48, 49].

The reported age range of MANs (46 cases) is 16 to 82 years, with a mean and median age of 51 and 51.5 years, respectively. In general, cases in females slightly outnumber those in males $(26: 20)$. Hormone hypersecretion occurs frequently in these tumors; only 13 out of 42 cases were non-functioning (eight adenomas, one borderline and four carcinomas), whereas five patients had biochemical evidence of hormone production (two adenomas, one borderline and two carcinomas). The remaining 24 cases with an available endocrine evaluation presented with clinical evidence of hormone overproduction: 16 with Cushing-related symptoms, 4 with Conn syndrome, 2 with symptoms due to cortisol and androgens, 1 with gynecomastia and breast pain and 1 with virilization (4 adenomas and 20 carcinomas). Although it had been suggested that the functional status could be associated with the presence of myxoid material, non-functioning tumors displaying a higher percentage of myxoid areas and being lipid depleted [61], no definite conclusions can be reached on morphological grounds. In fact, eight cases showing a myxoid area $>70 \%$ were hormonally active [48-50, 62, 63], whereas another eight cases with a similar myxoid content were non-functional [48, 51, 53-55, 57, 59, 60]. The proportion of myxoid change varied in all reported cases from $5 \%$ to approximately $100 \%$ of the total area. Interestingly, neurofilament (NF) expression has been recently found to be related more to the extent of myxoid changes and, therefore, suggested as a potential characteristic of myxoid ACCs with a predominant ( $>70 \%$ ) myxoid component [48]. This, along with the fact that the expression of neuroendocrine/neuronal markers such as $\mathrm{NF}$ and CD56 was mostly restricted to the myxoid areas of conventional ACCs with focal myxoid change, seems to imply a peculiar association between NF expression and myxoid change [48].

An important point is the distinction of myxoid borderline/malignant ACTs into two subgroups as proposed by Papotti et al. [48]: myxoid ACCs and conventional ACCs with focal myxoid changes. This is based on a different type of myxoid change, growth pattern, cell size, nuclear atypia and cell cytoplasm. Myxoid ACCs are usually characterized by (1) extensive myxoid areas separated from conventional ACC areas, if present, (2) trabecular/microacinar growth pattern, (3) small, uniform cell size, (4) mild nuclear atypia and (5) scant, eosinophilic cell cytoplasm, whereas conventional
ACCs with focal myxoid changes are characterized by (1) focal myxoid areas $(<20 \%$ of the total area) always merging with conventional ACC areas, (2) solid/diffuse growth pattern, (3) large, heterogenous cell size, (4) moderate to high nuclear atypia and (5) abundant, granular eosinophilic cell cytoplasm. Nevertheless, two cases of the former subset showed overlapping features such as solid growth pattern and pleomorphism as well. In addition, the same investigators [48] suggested that only the former subgroup actually belongs to the distinctive myxoid variant of ACTs, with the latter potentially representing the result of myxoid degenerative changes (Fig. 3). Whether or not these subgroups have a distinct biologic behavior needs to be further elucidated.

In the histopathological assessment of these lesions, it is critical first to exclude potential morphological mimics, including metastatic tumors affecting the adrenal gland (breast carcinomas, salivary or skin adnexal carcinomas of adenoid cystic type and neuroendocrine tumors) [48] as well as primary retroperitoneal neoplasms with myxoid areas (chordomas, myxomas, lipomas or liposarcomas, leiomyomas or leiomyosarcomas, benign or malignant nerve sheath tumors, extraskeletal myxoid chondrosarcomas, gastrointestinal stromal tumors and myxoid malignant fibrous histiocytomas) [49, 50,63]. The presence of typical foci of adrenocortical differentiation, along with an immunohistochemical profile similar to that of the conventional ACT, provides valuable aid in excluding these mimics [48, 50, 51]. In addition, accurate assessment of malignant potential in any given tumor of the myxoid variant is important. In this regard, the issue of whether the Weiss scoring system is adequate to reliably predict malignancy in MANs is currently being questioned because:

(1) A MAN case lacking morphological signs of malignancy (Weiss score 1/clear cell cytoplasm present in less than $25 \%$ of the tumor cells) and diagnosed as myxoid borderline ACT [59] subsequently did demonstrate an aggressive clinical behavior (peritoneal spread), with a dismal outcome 68 months following the initial diagnosis [48]. Another such tumor of uncertain malignant potential (Weiss score 1/clear cell cytoplasm present in less than $25 \%$ of the tumor cells) has been recently described by the same group, with no evidence of recurrence or metastasis 9 months following surgical resection. Both cases were characterized by the same extent of myxoid area (90\%), growth pattern, Weiss score and low Ki67 labeling index (3\% and 4\%, respectively). Interestingly, the latter case had an intact reticulin network as opposed to the former, which displayed stromal framework disruption as confirmed by the reticulin stain [48]. 
Fig. 3 Section of a myxoid adrenocortical carcinoma showing a necrotic area at the right of the field (a). Tumor cells are arranged in a small nested (b) or trabecular/cord-like (c) growth pattern focally displaying marked nuclear atypia $(\mathbf{b}-\mathbf{c})$. Area of conventional adrenocortical carcinoma $(60-70 \%$ of total area in histologic sections); neoplastic cells are arranged in an interanastomosing trabecular pattern showing severe nuclear atypia near the center of the field (d)

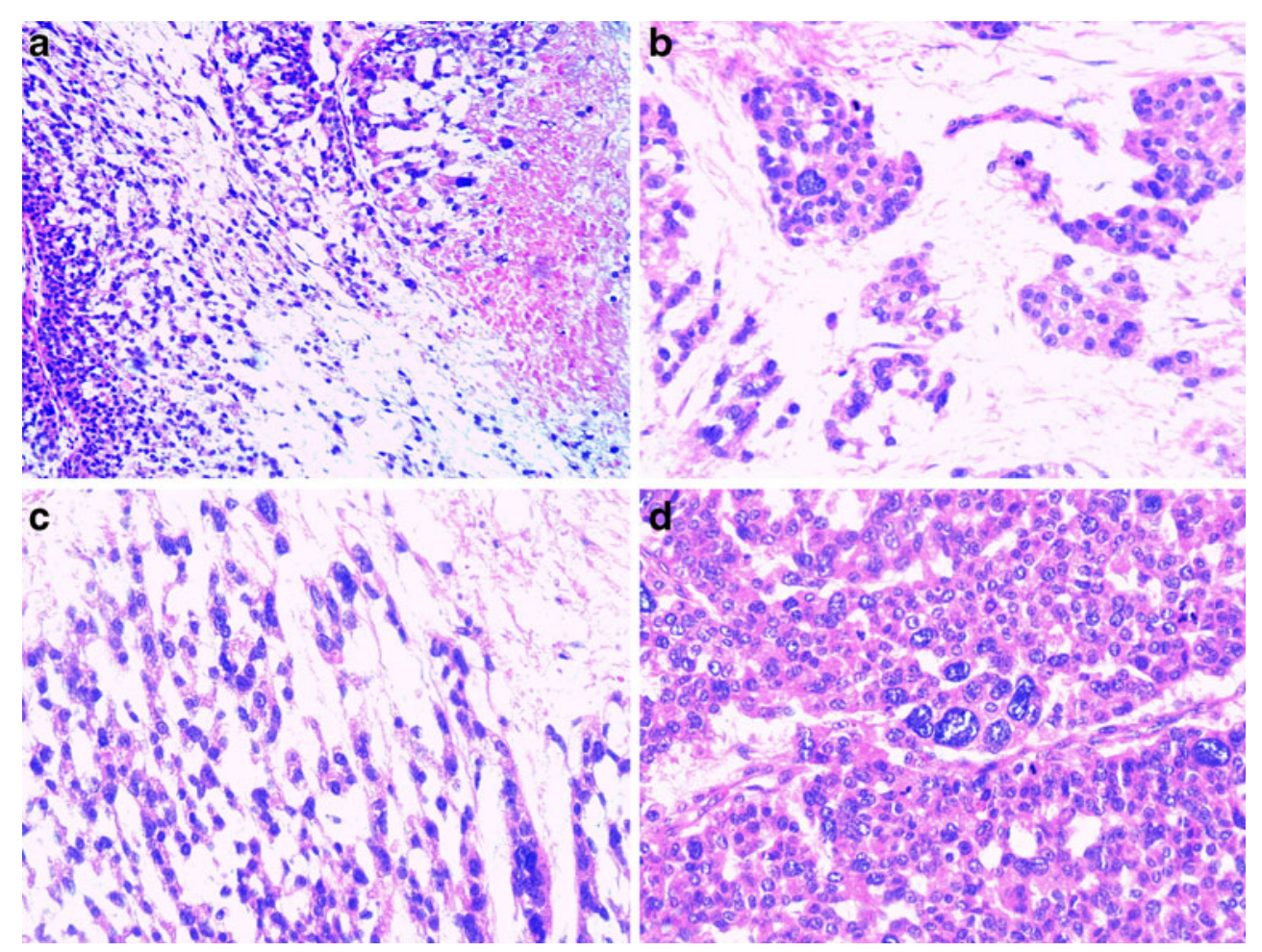

(2) Another MAN of borderline malignancy (average mitoses 3/10 HPFs, mild to moderate nuclear pleomorphism, cells with ample predominantly eosinophilic cytoplasm, occasional clear cells and a tumor weight of $94 \mathrm{~g}$ ) did not recur or metastasize after a follow-up period of 18 months [50]. In addition, four out of six myxoid ACAs reported in a series by Brown et al. [49] were large ( $\geq 6 \mathrm{~cm})$, showed focal capsular invasion and a mitotic activity of $\geq 1 / 10$ HPFs in the absence of recurrence or metastatic disease after a mean follow-up period of 12 years. It should be stressed that 9 out of 17 myxoid ACAs were relatively large $(\geq 6 \mathrm{~cm})$, a feature further challenging the prediction of a benign clinical course [49, 50, 54-57].

(3) Some of the Weiss score parameters were hard to assess in the myxoid areas, while others could be identified in a variable percentage according to observations made by Papotti et al. [48] in the subset of myxoid ACCs. In particular, diffuse growth pattern was never a feature, atypia was minimal or absent, and small vessel invasion was less apparent due to myxoid areas in contrast to necrosis, "dark" cell cytoplasm and mitotic figures which were the only readily apparent criteria. In keeping with this, Hsieh et al. [62] reported on a myxoid ACC showing an extensive myxoid area ( $80 \%$ of the tumor area) of Weiss score (3) and a nonmyxoid component of Weiss score (6); the latter consisted of three growth patterns (trabecular, solid and clear cell), each exhibiting different Weiss score parameters. Moreover, Brown et al. [49] noted that mitoses, when present, were generally less frequent in myxoid areas, while extensive extracapsular involvement was a feature only of carcinomas.

Adrenocortical carcinomas with a sarcomatous or sarcoma-like component

According to WHO classification 2004, oncocytic ACCs, myxoid ACCs and ACCs with sarcomatous areas are regarded as rare variants of ACC [66]. Whether the latter variant should be designated carcinosarcoma or sarcomatoid carcinoma, as previously suggested by Sturm and colleagues [67] in agreement with the WHO terminology applied to biphasic tumors with pleomorphic, sarcomatous or sarcomatoid elements arising in other organs [68], is still unsettled. Nevertheless, nine cases of this unusual variant have been previously reported $[67,69-76]$, with only four corresponding to true carcinosarcomas [69, 72-74], consistent with the concept that carcinosarcomas are tumors composed of morphologically malignant epithelial and specialized mesenchymal elements, easily recognizable histologically as forms of mesenchymal malignancy resembling osteosarcoma, chondrosarcoma, rhabdomyosarcoma, angiosarcoma or liposarcoma (Fig. 4) [77]. Adrenal carcinosarcomas, like their counterparts in other organ systems, were mainly characterized by a rhabdomyosarcomatous component (three out of four) $[69,73,74]$, while only one case was characterized by a mixture of osteosar- 
Fig. 4 Sections of an adrenocortical carcinosarcoma comprising a well-differentiated carcinomatous component (a) and a sarcomatous component demonstrating osteosarcomatous differentiation (b) along with areas of uniform spindle cells arranged in a fascicular (c) or herringbone (d) growth pattern

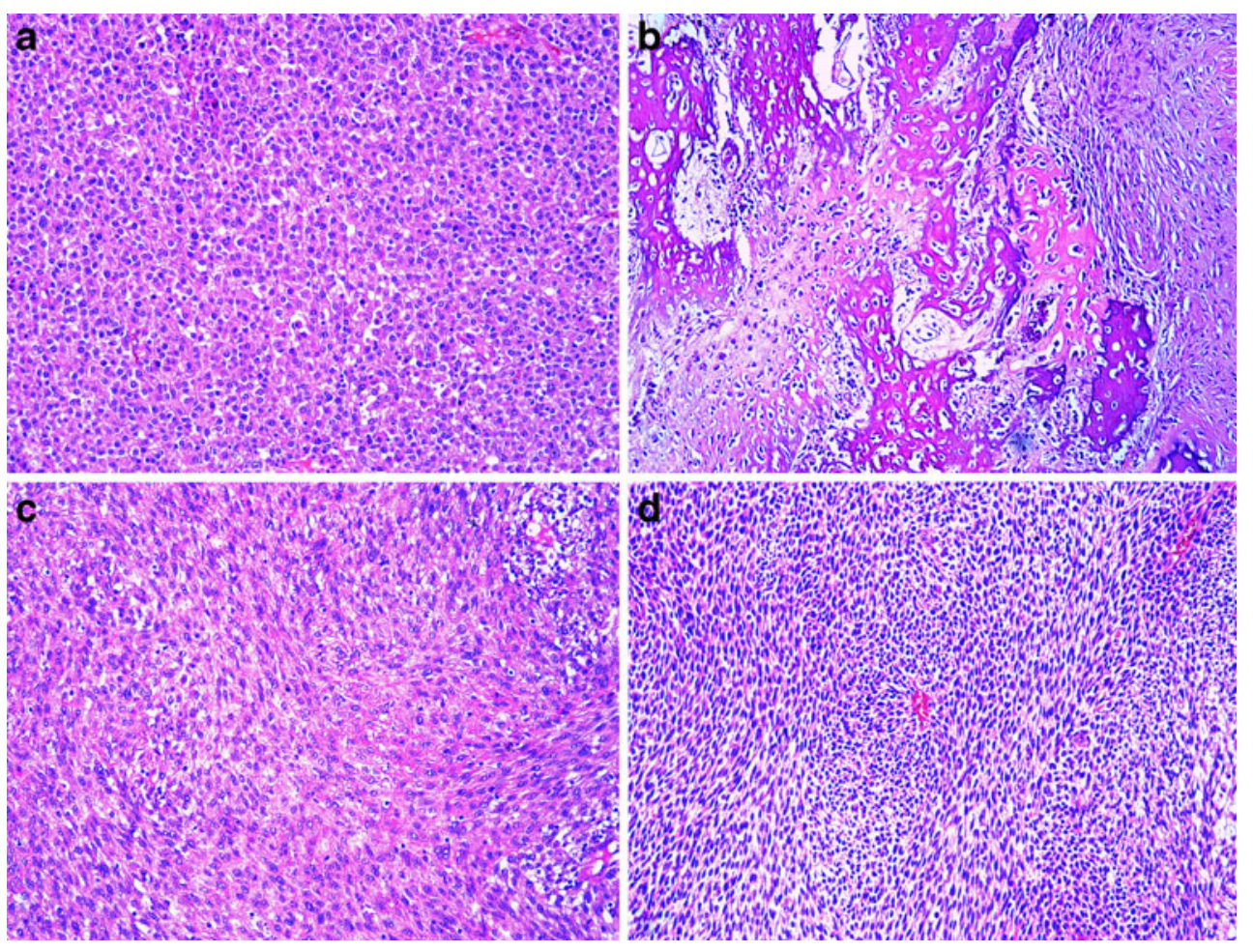

coma and chondrosarcoma [72]. The remaining five biphasic cases without heterologous elements did have a spindle cell component $[67,70,71,75,76]$, with three out of five also showing variably giant cells in the sarcomatous component [67, 70, 75].

Another unique malignant biphasic neoplasm arising as a primary tumor of the adrenal gland is the adrenocortical blastoma. This functional (virilizing) malignant tumor has been reported in an infant, and it consisted of a mixture of immature epithelial and mesenchymal elements, along with slit-like spaces partially lined by primitive epithelial cells, focally recapitulating the morphology of the normal embryologic development of the adrenal cortex [78]. There have been no additional cases reported, and therefore, the question whether this neoplasm is indeed restricted to the pediatric age group [79] similar to pleuropulmonary blastoma (virtually restricted) but in contrast to pulmonary blastoma [77] remains unresolved.

Of interest, only two out of four carcinosarcomas were functional, presenting with clinical signs either of hyperaldosteronism or of virilization [72, 73], whereas all adrenocortical sarcomatoid carcinomas were nonfunctioning [67, 70, 71, 75, 76].

Although the limited number of reported cases does not allow any definite conclusion, adrenocortical carcinosarcomas and sarcomatoid carcinomas have similar clinical outcome (postoperative survival ranging from 2 days to 12 months; median 6 months) [69] and therefore have a worse prognosis than their conventional counterparts [67]. This dramatically aggressive behavior may be attributed not only to advanced stage at presentation but also in part to inherent biological properties potentially deriving by progression (dedifferentiation) from a pre-existing better differentiated ACC, in analogy to the undifferentiated (anaplastic) carcinoma of the thyroid gland $[66,80]$. This endows cells with migratory and invasive properties, epithelial-mesenchymal transition, stemness, prevention of apoptosis and senescence, and it contributes to immunosuppression and notably confers resistance to chemotherapy and immunotherapy [81].

Although various theories have been proposed with regard to the histogenesis of carcinosarcomas, namely (1) the composition tumor theory (paradoxically requiring a nonmalignant non-epithelial component as a reactive proliferative response induced by the epithelial component via paracrine secretion), (2) the collision or biclonal tumor theory (a collision between two synchronous, histogenetically independent, biclonal tumors), (3) the conversion tumor theory (neoplastic transformation within a monoclonal tumor recapitulating the naturally occurring event of conversion of epithelial to mesenchymal cells during embryogenesis) [81] and (4) the combination or divergent tumor theory (deriving from a common monoclonal stem cell precursor) [82-85], molecular genetic evidence of monoclonality supports the single pluripotential stem-cell-divergence hypothesis and the epithelial-to-mesenchymal transition as well [83-85]. With regard to ACCs with sarcomatous areas, evidence emerging from the immunophenotypical profile of the sarcomatous component (focal retaining of Melan-A, synaptophysin and 
calretinin positivity) [69] and the presence of transitional (intermingled) zones between carcinomatous and sarcomatous components $[67,70,73]$ seem to imply such a putative transition. Differences of the mitotic count in the distinct elements [7/10 HPF (epithelioid component) versus 30/10 HPF (pleomorphic/spindle cell component)] [69] and the presence only of the sarcomatous component either in large veins into the periadrenal fat [67] or in well-established metastases [71, 74] are consistent with a dedifferentiation process.

Aberrant phenotypical differentiation observed in this particular variant includes both neural and melanocytic differentiation (NSE, S100 and HMB-45 immunoreactivity) [70, 71]. Although melanocytic differentiation, suggestive of a divergent evolutionary pathway, has been reported in carcinosarcomas of other anatomic locations [86-89], pathologists should always keep in mind to first exclude the possibilities of a metastatic melanoma, a collision tumor consisting of an ACC and a primary or metastatic melanoma (not yet reported) and a primary perivascular epithelioid cell tumor [90]. Other differential diagnostic considerations include composite pheochromocytoma with a malignant peripheral nerve sheath tumor component (the latter with or without divergent differentiation into mesenchymal elements such as rhabdomyo-, osteo-, chondro-, angio- and/or fibro-sarcomatous components) [91, 92], metastatic carcinoma with sarcomatous or sarcoma-like areas and metastatic neoplasm with sarcomatoid elements other than carcinomas (melanoma or germ cell tumor), as well as primary retroperitoneal sarcomas [69]. Given that adrenal biphasic tumors, in contrast to those arising in epithelial organs, are unlikely to express cytokeratins while focal smooth muscle differentiation is a common finding, thorough sampling of the resected tumor is of critical importance in order to reveal the co-existent carcinomatous component and subsequently prove the adrenal origin on immunohistochemical grounds [67, 69].

Since ACCs with sarcomatous areas are highly aggressive tumors and a sarcomatous or sarcomatoid component seems to be a predictor of shorter survival in ACC, the question arises whether the Weiss scoring system should be applied to this extremely rare ACC variant. In fact, ACCs with sarcomatous areas regroup the majority of Weiss histological criteria [67]. In this setting, Sturm and colleagues [67] have proposed that a sarcomatous or sarcomatoid component should be well circumscribed and represent at least $10 \%$ of the tumor bulk to establish this unusual diagnosis. Nevertheless, the sarcomatous or sarcomatoid component is not included in the Weiss system, further confounding this issue when dealing with a biphasic tumor demonstrating a predominant non-carcinomatous component [67, 69]; in such cases, the criterion of diffuse architecture (greater than 1/3) is being obviously challenged.

\section{Conclusion}

In summary, we discussed the issue of multistep tumorigenesis in adrenocortical neoplasia and provided an overview of the ACN variants in order to make histopathologists aware of the clinicopathological spectrum. It is important to correctly characterize their biological potential, which is occasionally not an easy task for myxoid and oncocytic adrenocortical tumors. In this context, applying the Weiss scoring system on the latter would definitely tilt the diagnostic balance towards malignancy in a similar way as pediatric ACNs (pathologically malignant but benign-behaving tumors) [8], whereas underdiagnosis could potentially be the case with regard to the myxoid ones. In this setting, even the multistep tumorigenic processes are hard to take apart and analyze, while evidence for a distinct biological behavior is either preliminary [39] or unestablished [48] due to the small number of reported cases, limited follow-up period, insufficient data and heterogeneity. Whenever pathologists report on these variants singly or as part of larger series, it is recommended to note the diagnostic subcategory, as has been previously suggested (pure oncocytic tumor/mixed oncocytic tumor/ordinary adrenocortical tumor with focal oncocytic changes, myxoid ACCs/conventional ACCs with focal myxoid changes and carcinosarcoma/ sarcomatoid carcinoma) [39, 48].

Conflict of interest statement We declare that we have no conflict of interest.

Open Access This article is distributed under the terms of the Creative Commons Attribution Noncommercial License which permits any noncommercial use, distribution, and reproduction in any medium, provided the original author(s) and source are credited.

\section{References}

1. Fassnacht M, Libe R, Kroiss M, Allolio B (2011) Adrenocortical carcinoma: a clinician's update. Nat Rev Endocrinol 7:323-335

2. McNicol AM (2011) Update on tumours of the adrenal cortex, phaeochromocytoma and extra-adrenal paraganglioma. Histopathology 58:155-168

3. Lau SK, Weiss LM (2009) The Weiss system for evaluating adrenocortical neoplasms: 25 years later. Hum Pathol 40:757-768

4. Aubert S, Wacrenier A, Leroy X et al (2002) Weiss system revisited: a clinicopathologic and immunohistochemical study of 49 adrenocortical tumors. Am J Surg Pathol 26:1612-1619

5. Blanes A, Diaz-Cano SJ (2007) Histologic criteria for adrenocortical proliferative lesions: value of mitotic figure variability. Am J Clin Pathol 127:398-408

6. Volante M, Bollito E, Sperone P et al (2009) Clinicopathological study of a series of 92 adrenocortical carcinomas: from a proposal of simplified diagnostic algorithm to prognostic stratification. Histopathology 55:535-543

7. Tissier F (2010) Classification of adrenal cortical tumors: what limits for the pathological approach? Best Pract Res Clin Endocrinol Metab 24:877-885 
8. Dehner LP, Hill DA (2009) Adrenal cortical neoplasms in children: why so many carcinomas and yet so many survivors? Pediatr Dev Pathol 12:284-291

9. Bertherat J, Bertagna X (2009) Pathogenesis of adrenocortical cancer. Best Pract Res Clin Endocrinol Metab 23:261-271

10. Grumbach MM, Biller BM, Braunstein GD et al (2003) Management of the clinically inapparent adrenal mass ("incidentaloma"). Ann Intern Med 138:424-429

11. Sidhu S, Marsh DJ, Theodosopoulos G et al (2002) Comparative genomic hybridization analysis of adrenocortical tumors. J Clin Endocrinol Metab 87:3467-3474

12. Gruschwitz T, Breza J, Wunderlich H, Junker K (2010) Improvement of histopathological classification of adrenal gland tumors by genetic differentiation. World J Urol 28:329-334

13. Kjellman M, Larsson C, Bäckdahl M (2001) Genetic background of adrenocortical tumor development. World J Surg 25:948-956

14. Gicquel C, Leblond-Francillard M, Bertagna X et al (1994) Clonal analysis of human adrenocortical carcinomas and secreting adenomas. Clin Endocrinol (Oxf) 40:465-477

15. Beuschlein F, Reincke M, Karl M et al (1994) Clonal composition of human adrenocortical neoplasms. Cancer Res 54:4927-4932

16. Kjellman M, Roshani L, Teh BT et al (1999) Genotyping of adrenocortical tumors: very frequent deletions of the MEN1 locus in $11 \mathrm{q} 13$ and of a 1-centimorgan region in 2p16. J Clin Endocrinol Metab 84:730-735

17. Bernard MH, Sidhu S, Berger N et al (2003) A case report in favor of a multistep adrenocortical tumorigenesis. J Clin Endocrinol Metab 88:998-1001

18. Trezzi R, Poli F, Fellegara G (2009) "Dedifferentiated" adrenal cortical neoplasm. Int J Surg Pathol 17:343-344

19. Gaujoux S, Tissier F, Groussin L et al (2008) Wnt/beta-catenin and 3',5'-cyclic adenosine 5'-monophosphate/protein kinase A signaling pathways alterations and somatic beta-catenin gene mutations in the progression of adrenocortical tumors. J Clin Endocrinol Metab 93:4135-4140

20. Schmitt A, Saremaslani P, Schmid S et al (2006) IGFII and MIB1 immunohistochemistry is helpful for the differentiation of benign from malignant adrenocortical tumours. Histopathology 49:298-307

21. van't Sant HP, Bouvy ND, Kazemier G et al (2007) The prognostic value of two different histopathological scoring systems for adrenocortical carcinomas. Histopathology 51:239-245

22. de Reyniès A, Assié G, Rickman DS et al (2009) Gene expression profiling reveals a new classification of adrenocortical tumors and identifies molecular predictors of malignancy and survival. J Clin Oncol 27:1108-1115

23. Giordano TJ, Kuick R, Else T et al (2009) Molecular classification and prognostication of adrenocortical tumors by transcriptome profiling. Clin Cancer Res 15:668-676

24. Laurell C, Velázquez-Fernández D, Lindsten K et al (2009) Transcriptional profiling enables molecular classification of adrenocortical tumours. Eur J Endocrinol 161:141-152

25. Ragazzon B, Libé R, Gaujoux S et al (2010) Transcriptome analysis reveals that p53 and \{beta\}-catenin alterations occur in a group of aggressive adrenocortical cancers. Cancer Res 70:8276-8281

26. Ragazzon B, Assié G, Bertherat J (2011) Transcriptome analysis of adrenocortical cancers: from molecular classification to the identification of new treatments. Endocr Relat Cancer 18:R15-R27

27. Bussey KJ, Demeure MJ (2009) Genomic and expression profiling of adrenocortical carcinoma: application to diagnosis, prognosis and treatment. Future Oncol 5:641-655

28. Bussey KJ, Demeure MJ (2011) Toward a pathway-centered approach for the treatment of adrenocortical carcinoma. Curr Opin Oncol 23:34-44

29. Martinerie C, Gicquel C, Louvel A et al (2001) Altered expression of novH is associated with human adrenocortical tumorigenesis. $\mathrm{J}$ Clin Endocrinol Metab 86:3929-3940
30. Berthon A, Sahut-Barnola I, Lambert-Langlais S et al (2010) Constitutive beta-catenin activation induces adrenal hyperplasia and promotes adrenal cancer development. Hum Mol Genet 19:15611576

31. Tissier F, Cavard C, Groussin L et al (2005) Mutations of betacatenin in adrenocortical tumors: activation of the Wnt signaling pathway is a frequent event in both benign and malignant adrenocortical tumors. Cancer Res 65:7622-7627

32. Tadjine M, Lampron A, Ouadi L, Bourdeau I (2008) Frequent mutations of beta-catenin gene in sporadic secreting adrenocortical adenomas. Clin Endocrinol (Oxf) 68:264-270

33. Bonnet S, Gaujoux S, Launay P et al (2011) Wnt/\{beta $\}$-catenin pathway activation in adrenocortical adenomas is frequently due to somatic CTNNB1-activating mutations, which are associated with larger and nonsecreting tumors: a study in cortisol-secreting and nonsecreting tumors. J Clin Endocrinol Metab 96:E419-E426

34. Tadjine M, Lampron A, Ouadi L et al (2008) Detection of somatic beta-catenin mutations in primary pigmented nodular adrenocortical disease (PPNAD). Clin Endocrinol (Oxf) 69:367-373

35. Gaujoux S, Grabar S, Fassnacht M et al (2011) $\beta$-catenin activation is associated with specific clinical and pathologic characteristics and a poor outcome in adrenocortical carcinoma. Clin Cancer Res 17:328-336

36. Durand J, Lampron A, Mazzuco TL, Chapman A, Bourdeau I (2011) Characterization of differential gene expression in adrenocortical tumors harboring \{beta\}-catenin (CTNNB1) mutations. J Clin Endocrinol Metab 96:E1206-E1211

37. Chapman A, Durand J, Ouadi L, Bourdeau I (2011) Identification of genetic alterations of AXIN2 gene in adrenocortical tumors. J Clin Endocrinol Metab (Epub ahead of print)

38. Kakimoto S, Yushita Y, Sanefuji T et al (1986) Non-hormonal adrenocortical adenoma with oncocytoma-like appearances. Hinyokika Kiyo 32:757-763

39. Wong DD, Spagnolo DV, Bisceglia M et al (2011) Oncocytic adrenocortical neoplasms - a clinicopathologic study of 13 new cases emphasizing the importance of their recognition. Hum Pathol 42:489-499

40. Saad R, Marsault S, Coloby P (2011) Adrenocortical oncocytic cells tumor: case report and review of literature. Prog Urol 21:288-290

41. Mwandila M, Waller H, Stott V, Mercer P (2010) A case of a testosterone-secreting oncocytic adrenocortical carcinoma. N Z Med J 123:80-82

42. Rosenkrantz AB, Do RK, Hajdu CH (2010) Imaging appearance of bulk fat within an oncocytic adrenocortical neoplasm, a rare and potentially malignant tumour. Br J Radiol 83:e204-e207

43. Cham E, Watkin W, Goldschmidt R, Liu L (2010) Fine needle aspiration cytology of adrenocortical oncocytic neoplasm: a case report. Acta Cytol 54:627-634

44. Ohtake H, Kawamura H, Matsuzaki M et al (2010) Oncocytic adrenocortical carcinoma. Ann Diagn Pathol 14:204-208

45. Bisceglia M, Ben-Dor D, Pasquinelli G (2005) Oncocytic adrenocortical tumors. Pathol Case Rev 10:228-242

46. Bisceglia M, Ludovico O, Di Mattia A et al (2004) Adrenocortical oncocytic tumors: report of 10 cases and review of the literature. Int J Surg Pathol 12:231-243

47. Willems SM, Wiweger M, van Roggen JF, Hogendoorn PC (2010) Running GAGs: myxoid matrix in tumor pathology revisited: what's in it for the pathologist? Virchows Arch 456:181-192

48. Papotti M, Volante M, Duregon E et al (2010) Adrenocortical tumors with myxoid features: a distinct morphologic and phenotypical variant exhibiting malignant behavior. Am J Surg Pathol 34:973-983

49. Brown FM, Gaffey TA, Wold LE, Lloyd RV (2000) Myxoid neoplasms of the adrenal cortex: a rare histologic variant. Am J Surg Pathol 24:396-401

50. Raparia K, Ayala AG, Sienko A, Zhai QJ, Ro JY (2008) Myxoid adrenal cortical neoplasms. Ann Diagn Pathol 12:344-348 
51. Dundr P, Novák K (2003) Pseudoglandular myxoid adenoma of the adrenal gland. Pathol Res Pract 199:493-496

52. Honda K, Kashima K, Daa T et al (2001) Myxoid adrenal cortical adenoma. Pathol Int 51:887-891

53. Jang KY, Chung MJ, Moon WS et al (2009) Adrenocortical adenoma with unusual myxoid histological pattern: a case report. Pathology 41:188-191

54. Ishida M, Yoshida K, Miyamoto K et al (2008) Cytological features of myxoid adrenocortical adenoma with a pseudoglandular component: a case report with differential diagnostic considerations. Diagn Cytopathol 36:576-580

55. Fine SW, Pindzola JA, Uhlman EJ, Epstein JI (2005) Pathologic quiz case: a 64-year-old man with an adrenal mass. Myxoid adrenal cortical neoplasm. Arch Pathol Lab Med 129:541-542

56. De Padua M, Rajagopal V (2008) Myxoid adrenal adenoma with focal pseudoglandular pattern. Indian J Med Sci 62:199-203

57. Zhu Y, Wu YX, Zhang CY et al (2008) Myxoid adrenocortical adenoma: a case report. Chin Med J (Engl) 121:1598-1600

58. Lu HS, Gan MF, Chen HS, Huang SQ (2008) Adrenal myelolipoma within myxoid cortical adenoma associated with Conn's syndrome. J Zhejiang Univ Sci B 9:500-505

59. Bollito ER, Papotti M, Porpiglia F et al (2004) Myxoid adrenocortical adenoma with a pseudoglandular pattern. Virchows Arch 445:414 418

60. Tang CK, Harriman BB, Toker C (1979) Myxoid adrenal cortical carcinoma: a light and electron microscopic study. Arch Pathol Lab Med 103:635-638

61. Forsthoefel KF (1994) Myxoid adrenal cortical carcinoma. A case report with differential diagnostic considerations. Arch Pathol Lab Med 118:1151-1153

62. Hsieh MS, Chen JH, Lin LW (2010) Myxoid adrenal cortical carcinoma presenting as primary hyperaldosteronism: case report and review of the literature. Int J Surg Pathol (Epub ahead of print)

63. Karim RZ, Wills EJ, McCarthy SW, Scolyer RA (2006) Myxoid variant of adrenocortical carcinoma: report of a unique case. Pathol Int 56:89-94

64. Suresh B, Kishore TA, Albert AS, Joy A (2005) Myxoid adrenal cortical carcinoma - a rare variant of adrenocortical carcinoma. Indian J Med Sci 59:505-507

65. Izumi M, Serizawa H, Iwaya K et al (2003) A case of myxoid adrenocortical carcinoma with extensive lipomatous metaplasia. Arch Pathol Lab Med 127:227-230

66. DeLellis RA, Lloyd RV, Heitz PU, Eng C (2004) World Health Organization classification of tumours. Pathology and genetics of tumours of endocrine organs. IARC, Lyon

67. Sturm N, Moulai N, Laverrière MH et al (2008) Primary adrenocortical sarcomatoid carcinoma: case report and review of literature. Virchows Arch 452:215-219

68. Travis WD, Brambilla E, Muller-Hermelink HK, Harris CC (2004) World Health Organization classification of tumours. Pathology and genetics of tumours of the lung, pleura, thymus and heart. IARC, Lyon

69. Sasaki K, Desimone M, Rao HR, Huang GJ, Seethala RR (2010) Adrenocortical carcinosarcoma: a case report and review of the literature. Diagn Pathol 5:51

70. Coli A, Di Giorgio A, Castri F (2010) Sarcomatoid carcinoma of the adrenal gland: a case report and review of literature. Pathol Res Pract 206:59-65

71. Lee MS, Park IA, Chi JG et al (1997) Adrenal carcinosarcoma-a case report. J Korean Med Sci 12:374-377

72. Barksdale SK, Marincola FM, Jaffe G (1993) Carcinosarcoma of the adrenal cortex presenting with mineralocorticoid excess. Am J Surg Pathol 17:941-945
73. Fischler DF, Nunez C, Levin HS et al (1992) Adrenal carcinosarcoma presenting in a woman with clinical signs of virilization. A case report with immunohistochemical and ultrastructural findings. Am J Surg Pathol 16:626-631

74. Decorato JW, Gruber H, Petti M, Levowitz BS (1990) Adrenal carcinosarcoma. J Surg Oncol 45:134-136

75. Collina G, Maldarizzi F, Betts CM, Eusebi V (1989) Primary sarcomatoid carcinoma of the adrenal gland. First case report. Virchows Arch A Pathol Anat Histopathol 415:161-167

76. Okazumi S, Asano T, Ryu M et al (1987) Surgical resection of adrenal carcinoma extending into the vena cava, right atrium and ventricle: case report and review of the literature. Nippon Geka Gakkai Zasshi 88:231-238

77. Weissferdt A, Moran CA (2011) Malignant biphasic tumors of the lungs. Adv Anat Pathol 18:179-189

78. Molberg K, Vuitch F, Stewart D, Albores-Saavedra J (1992) Adrenocortical blastoma. Hum Pathol 23:1187-1190

79. Lack EE (2007) Tumors of the adrenal glands and extraadrenal paraganglia—volume 8 (Afip Atlas of Tumor Pathology Series 4). American Registry of Pathology, Washington

80. Liu J, Brown RE (2010) Immunohistochemical detection of epithelialmesenchymal transition associated with stemness phenotype in anaplastic thyroid carcinoma. Int J Clin Exp Pathol 3:755762

81. Thiery JP, Acloque H, Huang RY, Nieto MA (2009) Epithelialmesenchymal transitions in development and disease. Cell 139:871-890

82. Guarino M, Tricomi P, Giordano F, Cristofori E (1996) Sarcomatoid carcinomas: pathological and histopathogenetic considerations. Pathology 28:298-305

83. Jin Z, Ogata S, Tamura $G$ et al (2003) Carcinosarcomas (malignant mullerian mixed tumors) of the uterus and ovary: a genetic study with special reference to histogenesis. Int J Gynecol Pathol 22:368-373

84. Dacic S, Finkelstein SD, Sasatomi E, Swalsky PA, Yousem SA (2002) Molecular pathogenesis of pulmonary carcinosarcoma as determined by microdissection-based allelotyping. Am J Surg Pathol 26:510-516

85. Thompson L, Chang B, Barsky SH (1996) Monoclonal origins of malignant mixed tumors (carcinosarcomas). Evidence for a divergent histogenesis. Am J Surg Pathol 20:277-285

86. Roma AA, Malpica A, Deavers MT (2011) Malignant melanoma arising in an ovarian carcinosarcoma: case report and review of the literature. Int J Gynecol Pathol 30:158-162

87. Liu J, Wu H (2011) Carcinosarcoma of female urethra with melanocytic differentiation. Int J Clin Exp Pathol 4:206-209

88. Amant F, Moerman P, Davel GH et al (2001) Uterine carcinosarcoma with melanocytic differentiation. Int J Gynecol Pathol 20:186-190

89. Kajo K, Zubor P, Spacek J, Ryska A (2007) Carcinosarcoma of the uterus with melanocytic differentiation. Pathol Res Pract 203:753758

90. Zarineh A, Silverman JF (2011) Adrenal perivascular epithelioid cell tumor: a case report with discussion of differential diagnoses. Arch Pathol Lab Med 135:499-502

91. Sakaguchi N, Sano K, Ito $M$ et al (1996) A case of von Recklinghausen's disease with bilateral pheochromocytomamalignant peripheral nerve sheath tumors of the adrenal and gastrointestinal autonomic nerve tumors. Am J Surg Pathol 20:889-897

92. Gupta R, Sharma A, Arora R, Vijayaraghavan M (2009) Composite phaeochromocytoma with malignant peripheral nerve sheath tumour and rhabdomyosarcomatous differentiation in a patient without von Recklinghausen disease. J Clin Pathol 62:659-661 